\title{
RETRACTED ARTICLE: Commentary on safety of outpatient admission and comparison of different surgical techniques in adult tonsillectomy
}

\author{
Kan-Feng Jin ${ }^{1} \cdot$ Zheng-Cai Lou ${ }^{2}$ (1)
}

Received: 25 February 2019 / Accepted: 4 March 2019 / Published online: 7 March 2019

(c) Springer-Verlag GmbH Germany, part of Springer Nature 2019

The editors have retracted this article [1] because it shows significant overlap with another publication by one of the co-authors [2]. This article [1] is therefore redundant. All authors agree to this retraction. The online version of this article contains the full text of the retracted article as electronic supplementary material.

\section{References}

1. Jin KF, Lou ZC (2019) Eur Arch Otorhinolaryngol. https://doi. org/10.1007/s00405-019-05368-X

2. Lou ZC, Lou ZH (2018) Post-tonsillectomy hemorrhage: underlying factors and prevention. Am J Otolaryngol 39(2):230-231

Electronic supplementary material The online version of this article (https://doi.org/10.1007/s00405-019-05368-x) contains supplementary material, which is available to authorized users.

Zheng-Cai Lou

louzhengcai@163.com

Kan-Feng Jin

jkangfeng@163.com

1 Department of Otorhinolaryngology, The Affiliated YiWu Hospital of Wenzhou Medical University, 322000 Yiwu City, Zhejiang Province, China

2 Department of Otorhinolaryngology, The Affiliated Yiwu Hospital, 699 Jiangdong Road, 322000 Yiwu City, Zhejiang Province, China 